\title{
L'APPRENTISSAGE DU DIALOGUE D'UN EXTRAIT DE FILM PAR DES APPRENANTS DE FLE : L'EFFET DU LEXIQUE DES EMOTIONS SUR LES PROCESSUS COGNITIFS DE MEMORISATION
}

\author{
Claire DEL OLMO \\ Université Toulouse 2 - Jean-Jaurès, France ; Université Lille 3 - Charles de Gaulle, France \\ claire.delolmo@univ-tlse2.fr ; claire.delolmo@univ-lille3.fr \\ Article reçu le 18 septembre 2017 | révisé depuis le 24 septembre 2017 | accepté le 14 décembre 2017 \\ RÉSUMÉ. Depuis 2010, les didacticiens spécialisés dans l'enseignement/apprentissage \\ des Langues Etrangères (LE) s'intéressent aux émotions. Nous suivons leur lignée. Dans \\ cet article, nous présentons une recherche qui s'inscrit en didactique cognitive des LE. \\ Nous appréhendons la manière dont les apprenants de Français Langue Etrangère \\ mémorisent les informations verbales d'un extrait de film, dont le dialogue contient des \\ mots du champ lexical des émotions. Afin d'observer ce phénomène, nous réalisons une \\ étude expérimentale auprès de 13 sujets chinois, de niveau B2 en français. Dans un \\ premier temps, les sujets apprennent le dialogue d'un extrait filmique afin de le \\ théâtraliser. Une semaine plus tard, nous leur demandons de rappeler le dialogue. Les \\ résultats montrent que les sujets mémorisent mieux les parties du dialogue qui sont \\ affectivement chargées, avec du lexique des émotions. Ces résultats ont des implications \\ claires quant à la création de matériel pédagogique ou la sélection de supports pour ceux \\ qui souhaitent optimiser les processus cognitifs de mémorisation des apprenants et \\ favoriser l'acquisition du vocabulaire, de structures linguistiques.
}

Mots-clés : cognition, dialogue, FLE, lexique des émotions, mémorisation

\begin{abstract}
Since 2010, the didacticians specialized on foreign language teaching are working on emotions. Our reflexion fits into this framework. This article presents a cognitive didactics research on foreign language. It deals with how learners memorize the dialogue of a film extract when this dialogue has emotional lexicon. In order to observe this phenomenon, an experimental study was conducted to 13 chinese subjects with a B2 level in french. First, the participants learn the dialogue of an extract in order to play the scene. A week later, they are asked to remember the dialogue. The results show that the participants memorize more the part of the dialogue with emotional lexicon. This results have clear implications for the creation of pedagogical tools and the selection of tools for people who want to improve the learner's cognitive memorization process and the acquisition of vocabulary and linguistic structures.
\end{abstract}

Keywords : cognition, french as a foreign language, emotional lexicon, memorization. 


\section{INTRODUCTION}

Les enseignants de Français Langue Etrangère (FLE) ont toujours utilisé des documents authentiques et cela même lorsqu'ils utilisaient des méthodologies anciennes, comme la traditionnelle méthodologie grammaire-traduction, en s'appuyant sur des textes littéraires de grands auteurs.

Les documents authentiques, produits par des membres de la communauté francophone et non par des didacticiens dans le but d'enseigner le FLE, ont plusieurs avantages. Comme l'indique "Internet et Enseignement des langues - FLE » (s.d.), ils représentent pour les professeurs un moyen infini de faire entrer des fragments de la réalité étrangère, de son actualité, dans la classe tout en répondant aux besoins linguistiques et culturels des apprenants.

Les documents authentiques constituent, de plus, pour les enseignants, un bon moyen d'exposer un usage réel de la langue française, des formes qui ne sont ni artificielles ni décontextualisées. Quand les enseignants les exploitent, cela leur permet d'exposer des discours en contexte (Beacco, 2000), qui reflètent la langue et la culture enseignées (Vergues, 2011).

Aussi, depuis les années 1980, avec la présence des magnétoscopes dans les salles de classe puis plus tard avec l'apparition d'internet et la présence des ordinateurs, il n'est pas rare que les enseignants de FLE exploitent les dialogues d'extraits de films de la langue-culture cible.

Pour Compte (1998), le document authentique audiovisuel, documentaire ou filmique, permet essentiellement les fonctions suivantes dans l'enseignement d'une LE :

- Illustration : Dans ce type de documents, la transmission d'un énoncé est toujours multimodale. L'apprenant entend comment l'énoncé est prononcé sur le plan verbal (constituants lexicaux, syntaxiques) et paraverbal (intonation, rythme, débit) mais il voit aussi comment la parole est gesticulée (mouvements du corps de l'énonciateur, qui transmet des significations et soutient la prosodie). Ce genre de documents peut donc servir de modèle aux apprenants pour faire une mise en situation, jouer une saynète, avoir une parole gesticulée, à la manière d'un natif.

- Médiation: Dans ces supports, les spectateurs accèdent facilement au sens grâce aux images, aux plans des réalisateurs ainsi qu'aux ambiances musicales et sonores. Les apprenants comprennent plus facilement un document audiovisuel qu'un document textuel.

- Facilitation: Ce genre de documents amène les apprenants à développer leurs connaissances sur la société étrangère, ses modes de vie, les représentations qu'on y trouve. Ce qui permet à ces derniers d'accroître leurs connaissances partagées avec les locuteurs de la langue qui leur est enseignée tout en transformant leurs représentations sur cette langue et la/les culture(s) qui l'accompagne(nt).

Aussi, depuis le milieu des années 2000, les didacticiens appréhendent le document authentique de nature cinématographique sous un nouvel angle: celui des émotions. Ils s'intéressent à la manière dont il procure $\mathrm{du}$ plaisir aux apprenants (Vianin, 2006), précisent comment les émotions qu'il leur provoque les motivent (Maury, 2012), et indiquent qu'il constitue un excellent moyen de leur présenter les normes culturelles de l'expression des affects (Mrowa-Hopkins et Strambi, 2008).

Nous nous inscrivons dans la lignée des réflexions de ces didacticiens. Nous nous intéressons à la dimension émotionnelle $\mathrm{du}$ document authentique de nature cinématographique et, plus précisément, à la question de la mémorisation du support filmique qui a un contenu émotionnel. Dans cet article, nous présentons une étude sur l'apprentissage du FLE à travers le cinéma, qui présente bien souvent de grandes scènes émotionnelles, en nous intéressant aux émotions représentées dans les films et posées verbalement par les acteurs (Bordwell et Thompson, 2009). Nous souhaitons comprendre de quelle manière les apprenants de FLE mémorisent le dialogue d'un extrait filmique, lorsque celui-ci 
présente une scène dont le dialogue contient des termes relevant du lexique des émotions.

Afin de comprendre comment les apprenants de FLE mémorisent le dialogue d'un extrait de film contenant des unités lexicales $\mathrm{du}$ champ des émotions, nous posons notre réflexion en Didactique Cognitive (Billières et Spanghero, 2005) des Langues Etrangères (LE). Nous passons en revue des études émanent du domaine de la psychologie cognitive, ou s'y référant, pour appréhender le fonctionnement des processus cognitifs impliqués dans la mémorisation du matériel verbal correspondant à/contenant du lexique des émotions.

Ces études mettent en lumière un phénomène particulier: en général, l'être humain mémorise mieux parmi le matériel verbal tout venant: les items ou structures qui sont affectivement chargés. Plusieurs recherches témoignent que le matériel verbal, lexical ou textuel, ayant une coloration émotionnelle est généralement mieux mémorisé que le matériel verbal neutre.

L'expérience de Doerkenson et Shimamura (2001, p. 7) montre, par exemple, l'impact de la signification émotionnelle qu'a un mot au niveau de sa mémorisation. Cette étude indique que les individus se rappellent mieux des mots qu'ils perçoivent comme plaisants ou désagréables. Ils stockent davantage dans leur Mémoire à Long Terme (MLT) les mots émotionnels, qu'ils soient positifs ou négatifs, que les mots neutres (ni plaisants ni désagréables).

Ces résultats sont corroborés par l'expérience de Wang et Fu (2011). Dans celle-ci, réalisée à partir de mots chinois, les participants retiennent mieux les mots quand ils sont négatifs ou positifs. Une semaine après avoir appris une liste de mots chinois, ils se rappellent mieux des mots émotionnels que des mots neutres, et cela de manière significative.

Doerkenson et Shimamura (2001) montrent par ailleurs que des informations visuelles (des couleurs dans leur étude) sont mieux mémorisées si elles sont présentées en même temps que des mots émotionnels, comparé à des mots neutres. Les mots émotionnels retiennent plus l'attention des individus. Lorsque des mots sont émotionnels, ils guident $\mathrm{l}^{\prime}$ «attention sélective », engendrant une perception délimitée, qui permet aux sujets d'acquérir de nouvelles connaissances parmi un flux infini d'informations (Ruph, 2011, p.32). Ce que nous mémorisons est déterminé par ce que nous percevons, notre perceptiondépendant elle-même de notre attention (Lair, 1997, p.12), liée à la charge émotionnelle des situations.

En sus, comme l'indique Legros (1990), lorsqu'un texte comprend des mots avec une connotation affective, les lecteurs s'en rappellent mieux. Dans sa recherche, entre la phrase «Une bande de surexcités à dérangé Mr Germain dans le vieil ascenseur malpropre » et « Une bande agitée a provoqué Mr Germain dans le vieil ascenseur crasseux »: les lecteurs retiennent mieux le second énoncé (Legros, 1990, p. 5). Ils se rappellent aussi mieux d'un texte lorsqu'un climat émotionnel est instauré dès le début.

Et cet effet du contexte émotionnel sur la mémorisation se retrouve aussi dans l'étude de Chabrol et Camus-Malavergne (1994, p. 9), où on voit comment un discours politique, présent dans la presse, est mieux retenu par ceux qui le reçoivent lorsque l'énonciateur s'implique personnellement mais aussi émotionnellement envers ce qu'il énonce. Plus un discours est expressif et a une touche émotionnelle, plus l'énonciateur le prend en charge sur le plan individuel et subjectif: plus les récepteurs s'en rappellent (Chabrol \& Camus-Malavergne, 1994, p. 15).

Cette revue de la littérature nous conduit à formuler les hypothèses suivantes :

- Hypothèse générale (sur les rapports entre mémoire et matériel verbal émotionnel) : les individus mémorisent mieux les données verbales quand elles sont chargées au niveau affectif.

- Hypothèse opérationnelle (dans le domaine de l'enseignement/apprentissage du FLE) : lorsqu'un enseignant exploite le dialogue d'un extrait de film français, les apprenants mémorisent d'avantage le lexique des émotions présent dans celui-ci et les énoncés qui comprennent ce type de lexique que les autres lexies et énoncés. 


\section{MÉTHODE}

\section{Participants}

Pour tester notre hypothèse opérationnelle, nous mettons sur pied une expérience. Cette expérience a lieu en 2013 au sein d'une université, en France, et se déroule en deux temps. 28 sujets chinois adultes $y$ participent. Ils ont un niveau B2 en français d'après le Conseil de l'Europe (2001) et comprennent tout document de nature audiovisuelle en français standard. Nos résultats concernent 13 sujets, soit ceux ayant participé aux deux temps de notre expérience.

\section{Déroulement}

Dans le premier temps, les sujets participent à une classe de FLE dans des groupes de 5 à 9 apprenants. Ils visionnent un extrait du film intitulé Les nuits de la pleine lune (00:29:46-00:31:08) d'Eric Rohmer et apprennent la scène pour la jouer. Pour cela chaque apprenant regarde la scène autant de fois qu'il le nécessite sur un ordinateur. Ensuite les participants réalisent une lecture interprétée du dialogue. Puis ils jouent la scène deux fois comme s'ils étaient les personnages $\mathrm{du}$ film. Ils jouent une fois chaque personnage.

Ensuite, Une semaine plus tard, 13 sujets réalisent un test de rappel, dit « libre » de l'extrait. Ils doivent rapporter le dialogue sans aucun indice. Ils font ensuite un test de rappel dit «indicé » du dialogue, avec des images de l'extrait servant d'indices de récupération des informations stockées dans leur mémoire et leur facilitant la tâche de rappel.

\section{Matériel}

Voici le dialogue de l'extrait de film de cette expérience, avec en italique les termes appartenant au lexique des émotions, identifiés grâce au logiciel d'analyse automatique de ce type de lexique, nommé Emotaix-Tropes (Piolat et Bannour, 2009).

Femme : -Je veux dire pardon pour tout à l'heure. J'ai été désagréable, je sais.

Homme : -Ne commençons pas à discuter maintenant, hein ? Va te coucher que l'un de nous deux dorme au moins.
Femme : -Bon, bonsoir. Mais je suis désolée.

Homme : -Désolée ? Tu ne sais dire que ça! Si tu es désolée, sois désolée. Si tu ne l'es pas, ne le dis pas, hein ? Sois franche. Tu es parfaitement contente qu'il y ait au moins quelqu'un qui soit content.

Femme : - Mais j'aimerais que toi tu sois content.

Homme : - Oui, c'est peut-être un peu beaucoup me demander. Mais si tu veux que je sois content et ben je suis content! Je suis très content! Je suis heureux ! Je suis un imbécile heureux! Si je veux vivre dans mon idiotie, ça me regarde. Va te coucher. Tu as peur? Rassure-toi : j'aipas envie de te battre. Si j'ai envie de battre quelqu'un, c'est moi, l'idiot, le crétin, le débile!

\section{Méthodologie d'analyse}

Pour observer comment les sujets mémorisent le dialogue, nous comparons la manière dont ils rappellent au deuxième temps de l'expérience (temps 2) :

- Les mots lexicaux relevant du lexique des émotions vs les autres types d'unités lexicales.

- Les mots lexicaux des énoncés comprenant une ou plusieurs unité(s) lexicale(s) appartenant au lexique des émotions vs les mots des énoncés qui n'en contiennent pas.

- Les énoncés avec une/des unité(s) lexicale(s) du lexique des émotions vs les énoncés sans.

Pour le rappel d'un mot lexical (Sahraoui, 2009, p. 186), nous attribuons : 2 points pour un rappel correct ; 1,75 points si un verbe est bien rappelé mais est mal conjugué ; 1,75 points si un pluriel est donné pour un singulier ; 1,75 points pour l'adverbe qui est rappelé seulement en partie ; 1 point si le sujet donne un synonyme; 0,5 point pour un mot de la même famille ; 0 point au mot non rappelé ou rappelé avec un autre mot lexical.

Nous octroyons, pour le rappel d'un mot grammatical (Sahraoui, 2009, p. 186) : 2 points pour un bon rappel ; 1,75 points quand le sujet a commis une faute de genre ou bien de nombre; 1,5 points quand il y a une erreur en même temps de genre et de nombre; 1 point si le sujet donne un mot 
lexical de sens équivalent; 0 point si le mot n'est pas rappelé ou rappelé avec un autre mot grammatical.

Afin de constater si les différences entre les scores moyens obtenus aux tests de rappel (libre et indicé) sont significatives, nous recourrons au test $t$ des étudiants. Lorsqu'il donne un $p$ inférieur à 0,05 cela signifie que la différence qu'il existe entre deux moyennes de scores est statistiquement significative.

\section{RÉSULTATS ET DISCUSSION \\ Principaux résultats du rappel libre}

La figure 1 illustre les résultats obtenus par les 13 sujets chinois au test de rappel libre (sans aucun indice) du dialogue.

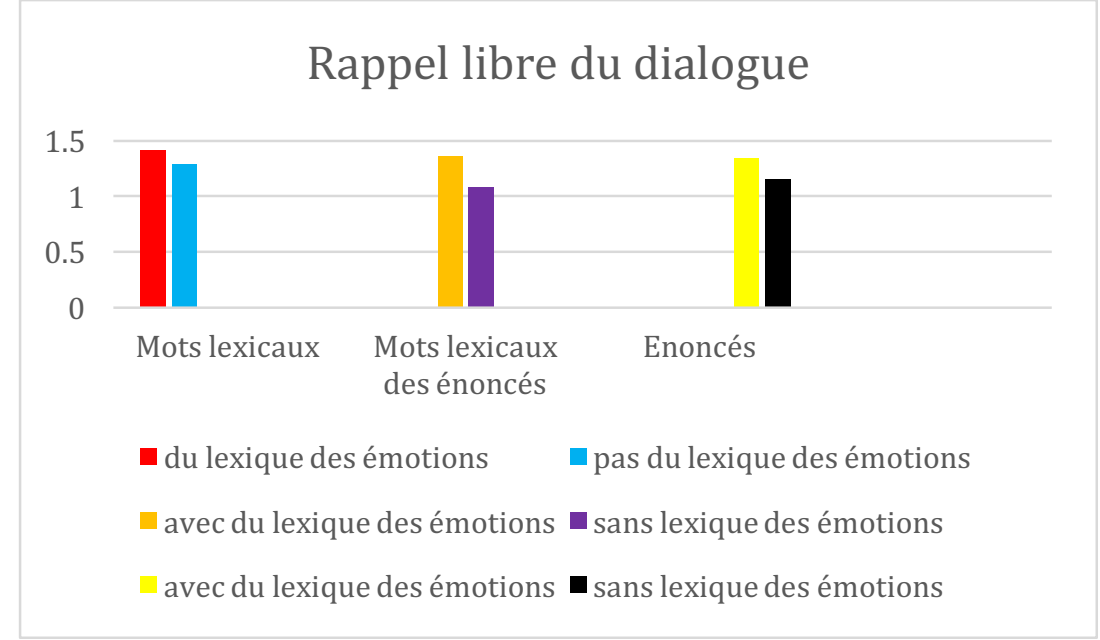

Figure 1. Rappel libre du dialogue au temps 2 de l'expérience.

Comme nous pouvons le constater avec cette figure, les sujets qui réalisent le test de rappel libre du dialogue de l'extrait semblent avoir mieux mémorisé les unités appartenant au lexique des émotions ainsi que les énoncés affectivement chargés, comprenant une/des unité(s) lexicale(s) du champ des émotions.

\section{Rappel des mots lexicaux}

Les sujets ont une moyenne de 1,41 pour le rappel des unités lexicales relevant du lexique desémotions (comme «envie») tandis qu'ils ont une moyenne de 1,28 pour le rappel des autres mots lexicaux (comme «demander»). Or il y a une différence statistiquement significative entre ces deux moyennes $(t(12)=2,666, p=0,021)$.

\section{Rappel des mots lexicaux des énoncés}

Les participants ont un score de 1,36 pour le rappel de l'ensemble des mots lexicaux des énoncés comprenant un ou plusieurs item(s) du lexique des émotions alors qu'ils ont un score valant 1,08 pour le rappel des mots lexicaux des énoncés qui ne comprennent pas ce genre d'items. La différence entre ces deux moyennes est statistiquement significative $(\mathrm{t}(12)=4,247, p=$ 0,001).

\section{Rappel des énoncés dans leur totalité}

Les sujets ont un score de 1,34 pour le rappel total (mots lexicaux et mots grammaticaux) des énoncés contenant une/des unité(s) lexicale(s) appartenant au lexique des émotions et un score valant 1,15 pour le rappel des énoncés sans ce type de lexique. Or ces scores sont significativement différents $(\mathrm{t}(12)=2,717, p=0,019)$.

\section{Principaux résultats du rappel indicé}

La figure 2 montre les résultats obtenus par les sujets au test de rappel indicé $\mathrm{du}$ dialogue (test comprenant des images de l'extrait facilitant la tâche rappel). 


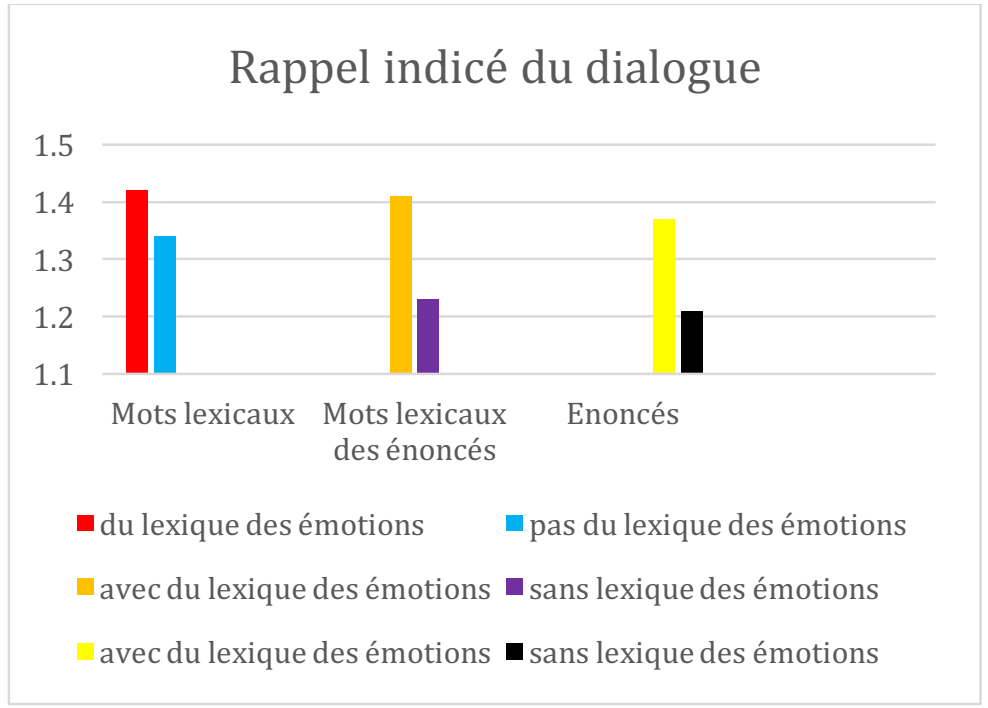

Figure 2. Rappel indicé du dialogue au temps 2 de l'expérience.

Comme nous pouvons le constater avec la figure 2, les sujets semblent aussi mieux rappeler le matériel verbal affectivement chargé au test de rappel indicé.

Pour ce test indicé, les sujets ont score moyen de 1,42 pour le rappel des mots lexicaux du lexique de l'affect tandis qu'ils n'obtiennent qu'un score de 1,34 pour les unités lexicales d'autreschamps. Ils ont un score de 1,41 pour le rappel des mots des énoncés comprenant du lexique de l'affect contre un score de 1,23 pour le rappel des mots des énoncés sans ce type de lexique. Puis ils ont un score de 1,37 pour le rappel des énoncés avec ce type de lexique contre un score de 1,21 pour le rappel des autres énoncés.

Il y a ici une différence statistiquement significative entre le rappel des mots lexicaux des énoncés comprenant du lexique de l'affect et celui des mots lexicaux des énoncés qui n'en comprennent pas $(\mathrm{t}(12)=-2,708, \mathrm{p}=$ $0,019)$.

Comme le rappel est indicé, facilité par des images de l'extrait, il est normal que les sujets rappellent les données verbales émotionnelles et non émotionnelles avec moins d'écart. Les images facilitent la récupération des informations stockées en mémoire. Cependant, nous pouvons ici remarquer que même lorsque les sujets ont des indices de récupération, ils se rappellent mieux du vocabulaire des énoncés comprenant du lexique des émotions que du vocabulaire des autres énoncés.

\section{Discussion des résultats}

Les résultats que nous obtenons confirment notre hypothèse de recherche. Lorsque des apprenants de FLE apprennent le dialogue d'un extrait de film pour le théâtraliser, ils retiennent d'avantage les données verbales qui sont chargées sur le plan de l'affect (soit les mots appartenant au lexique des émotions et les énoncés comprenant ce type d'unités lexicales).

Les apprenants stockent mieux dans leur MLT :

- les unités lexicales appartenant au lexique des émotions que les autres types de mots.

- Le vocabulaire des énoncés avec une/des unité(s) lexicale(s) appartenant au lexique des émotions que le vocabulaire des énoncés qui ne comprennent pas ce type de lexie.

- les énoncés (dans leur ensemble avec tous leurs mots lexicaux et grammaticaux) qui comprennent une/des unité(s) lexicale(s) relevant du champ des émotions que les énoncés qui n'en contiennent pas.

Cette expérience montre comment le contenu affectif d'un dialogue, en termes d'unités lexicales appartenant au champ de l'affect, influe sa mémorisation. Elle vient soutenir les résultats de nombreuses études à propos de la manière dont nous mémorisons les données de nature verbale en fonction de leur caractère émotionnel. Elle corrobore, par exemple, ceux des études que nous présentons au début de ce papier, comme 
celle de Wang et Fu (2011), indiquant que les mots émotionnels chinois sont mieux mémorisés que les mots neutres.

Cette étude complète, par ailleurs, les résultats d'autres recherches menées plus spécifiquement dans le champ de l'enseignement/apprentissage des LE.

Tel que Ghali et Frasson (2010) l'indiquent, les apprenants qui travaillent sur ordinateur via un logiciel apprennent mieux le vocabulaire lorsqu'ils éprouvent des émotions. Aussi, nous montrons, avec cette recherche, que l'acquisition du vocabulaire est optimisée lorsque les unités lexicales que l'on souhaite enseigner sont situées dans des énoncés qui sont affectivement chargés (qui comprennent une ou plusieurs unité(s) lexicale(s) relevant du lexique des émotions). Les apprenants apprennent mieux le vocabulaire s'il est inséré dans un contexte sémantique affectif. Plus le matériel verbal est affectif, plus il est mémorisé.

Puis si Cavalla (2006) souligne que l'un de nos devoirs en tant qu'enseignant de FLE est d'amener les apprenants à être capables d'exprimer leurs sentiments dans la langue cible, de leur enseigner le vocabulaire et les expressions idiomatiques servant à parler de leurs affects, car l'un des besoins premiers d'une personne est de se dire/de parler d'elle, nos résultats indiquent une qualité remarquable du lexique de l'affect. Ce type de lexique optimise la mémorisation de tournures linguistiques, d'unités lexicales et de mots grammaticaux qui s'inscrivent dans tous les champs.

Enfin, d'un point de vue plus épistémique, notre étude nous permet de souligner qu'il peut être intéressant d'appréhender l'usage didactique que nous pouvons avoir des émotions, la cognition et les processus de mémorisation étant déterminés par les dimensions émotionnelles des situations auxquelles nous faisons face. Elle nous permet de souligner que les études s'inscrivant dans le domaine de la Didactique Cognitive des LE; qui analysent les processus cognitifs à l'origine de la mémorisation de textes, de l'acquisition du vocabulaire et de structures langagières; gagneraient à considérer la charge émotionnelle du matériel verbal qui sert de support à l'apprentissage. Ce qui nous conduit à penser qu'il peut être pertinent de proposer un nouveau paradigme de recherche en lien avec celui de la Didactique Cognitive : celui de la Didactique CognitivoEmotionnelle des LE. Et cela afin de considérer les processus cognitifs dans leur complexité, en les appréhendant avec un regard plus global sous l'angle de leurs liens avec les émotions, comme le font les études menées dans le champ de la psychologie depuis plusieurs décennies.

\section{CONCLUSION}

Avec cette étude, nous validons notre hypothèse, s'intéressant aux processus cognitifs de mémorisation des apprenants de FLE: quand ces derniers apprennent le dialogue d'un extrait de film dans le but de le théâtraliser, ils mémorisent mieux les parties $\mathrm{du}$ dialogue comprenant du lexique des émotions. Ils se rappellent mieux: 1) des mots qui appartiennent au champ de l'affect que des autres types de mots; 2) des mots présents dans les énoncés qui comprennent $\mathrm{du}$ lexique des sentiments que des mots présents dans les autres énoncés; 3) des énoncés avec du lexique de l'affect que des énoncés sans. Ils mémorisent mieux le matériel verbal affectivement chargé que celui qui n'a pas de coloration émotionnelle.

Sur le plan pratique, notre étude nous permet de constater dans quelle mesure il peut être intéressant d'appréhender le contenu émotionnel $\mathrm{du}$ document avec lequel on travaille en classe de FLE. La constitution de ce dernier, en termes d'items lexicaux relevant du lexique des émotions, détermine la nature des acquisitions linguistiques des apprenants. Lorsque les apprenants apprennent le dialogue d'un extrait de film, ils stockent mieux dans leur MLT les parties comprenant ce genre de lexique. Ce qui nous donne de précieuses indications, pour favoriser l'acquisition de vocabulaire et de structures langagières, lorsque nous créons du matériel pédagogique ou que nous sélectionnons nos supports.

Enfin au niveau épistémique, notre recherche indique, sous un angle singulier, les liens existants entre matériel émotionnel 
et mémorisation de données verbales dans le champ du FLE. Ce qui nous incite à inviter les didacticiens à passer d'une Didactique Cognitive à une Didactique CognitivoEmotionnelle des LE. Et cela afin de considérer les processus cognitifs impliqués dans l'apprentissage des LE en ayant une approche plus globale de la cognition, en la considérant au regard des émotions, ces deux phénomènes étant indéniablement liés.

\section{REMERCIEMENTS}

Cette recherche a été menée en collaboration avec le Professeur Michel Billières que nous remercions vivement. Nous remercions également le laboratoire Octogone-Lordat, de 1'Université Toulouse 2 Jean-Jaurès, où a été effectuée notre expérience, ainsi que tous les sujets chinois qui y ont participé.

\section{RÉFÉRENCES}

Beacco, J.-C. (2000). Les dimensions culturelles des enseignements de langue: des mots aux discours. Paris, France : Hachette

Billières, M., \& Spanghero-Gaillard, G. (2005). La didactique cognitive des langues: regards croisés de disciplines pour comprendre le «comment». Revue Parole, 34, 101-136.

Bordwell, D. et Tompson. (2009). L'art du film: une introduction. Bruxelles, Belgique : De Boeck Université.

Cavalla, C. (2006). Lexique et représentation des sentiments. Dans V. Louis, N. Auger et I. Belu (dir.), Former les professeurs de langues à l'interculturel. A la rencontre de différents publics (p.185198). Louvain-la-Neuve, Belgique : Cortil-Wodon : E.M.E

Chabrol, C., \& Camus-Malavergne, O. (1994). Un discours politique en réception: mémorisation et compréhension. Mots, 40, 7-24.

Compte, C. (1998). Un document télévisuel pour parfaire l'apprentissage des langues? Dans M. Letzelter et F.-J. Meissner (dir.), Actes du Congrès de Tours L'enseignement des deux langues partenaires (vol.2, p.613-624). Tübingen, Allemagne : Gunter Narr Verlag.
Conseil de l'Europe. (2001). Cadre européen commun de référence pour les langues: apprendre, enseigner, évaluer. Paris, France : Didier.

Doerksen, S. et Shimamura, P. (2001). Source memory enhancement for emotional words. Emotion, 1(1), 5-11.

Ghali, R. et Frasson, C. (2010). Emotional strategies for vocabulary learning. Proceedings of the 10th IEEE International conference on advanced learning technologies (p.249-253). IEEE.

[Internet et Enseignement des langues FLE]. (s.d.). Repéré à http://flenet.unileon.es/theor2.htm

Lair, S. (1997). Savoir maîtriser sa mémoire. Paris, France : Retz.

Legros, D. (1990). Dramatisez... il en restera toujours quelque chose... . Glossa, Les Cahiers de l'Unadrio, 20, 4-9.

Maury, C. (2012). L'intégration du cinéma hollywoodien en classe de langue: réflexions sur quelques points de convergence disciplinaires. Recherche et pratiques pédagogiques en langues de spécialité, 31(2), 26-43.

Mrowa-hopkins, C. et Strambi, A. (2008). La dimension émotionnelle de la communication en situation interculturelle: l'expression non verbale de la colère chez des locuteurs angloaustraliens, français et italiens. Les Cahiers de l'Acedle, 3, 89-113.

Piolat, A. et Bannour, R. (2009). EMOTAIX : un scénario de Tropes pour l'identification automatisée du lexique émotionnel et affectif. L'Année Psychologique, 109, 655-698.

Vergues, M. (2011). Peut-on prétendre développer la compétence interculturelle au niveau faux débutant? Enjeux pour le didacticienpédagogue. Dans H. De Fontenay (dir.), Classe de langues et culture(s): vers l'interculturalité? Actes du 9e Colloque international de l'AFDECE (p. 325-254). Paris, France : L'Harmattan.

Sahraoui, H. (2009). Contribution à l'étude des stratégies compensatoires dans l'agrammatisme. Approche neuropsycholinguistique de la performance de six locuteurs agrammatiques en 
production orale: caractérisation quantitative et fonctionnelle des variabilités (Thèse de doctorat inédite). Université de Toulouse 2 Jean-Jaurès.

Ruph, F. (2011). Guide de réflexion sur les stratégies d'apprentissage à l'université (2e ed.). Québec, Canada : Presses de l’Université du Québec.

Vianin, P. (2006). La motivation scolaire: comment susciter le désir d'apprendre?
Bruxelles, Belgique : De Boeck \& Larcier.

Wang, B. et Fu, X. (2011). Time course of effects of emotion on item memory and source memory for Chinese words. Neurobiology of Learning and Memory, 95(4), 415-424. 